le esperienze che confermano l' esistenza delle deformazioni di un dielettrico posto in un campo elettrico, e quelle intraprese per verificare l'eguaglianza fra la costante dielettrica di una sostanza ed il quadrato del suo indice di rifrazione, l'Autore descrive le esperienze che si riferiscono alla penetrazione delle ondulazioni elettriche nei conduttori, alle azioni elettrodinamiche dei coibenti, alla propagazione ondulatoria del magnetismo ed alla misura della velocità di propagazione delle ondulazioni elettriche in varii mezzi e termina con quella parte che potrebbe chiamarsi l' ottica con onde elettriche nella quale sono descritte le esperienze che, riproducendo ad uno ad uno coi raggi di forza elettrica i fenomeni prodotti dai raggi luminosi, offrono una cosi brillante verifica esperimentale alla teoria elettromagnetica della luce.

Neglf ultimi due capitoli l'A. tratta degli studi fatti per determinare la durata di vibrazione degli eccitatori e risonatori hertziani, di Lecher e di Blondlot, e svolta, nei suoi punti fondamentali, la teoria di Bjerknes sulla risonanza, deșcrive le esperienze fatte dal Bjerknes stesso e da altri a verifica di questa teoria, e termina con una succinta descrizione delle esperienze del Garbasso sull' assorbimento, riflessione e dispersione delle onde elettriche effettuate da masse di risonatori elettrici da essi colpiti, mostrando l' appoggio che queste esperienze possono offrire all' interpretazione data dal Sarasin e de la Rive al fenomeno della risonanza multipla.

A completare l'utilità dell'opera è premesso alla medesima un diligentissimo catalogo, in ordine cronologico, delle opere e memorie scientifiche relative all' argomento.

Prof. D. MazzotTo.

\title{
ELEMENTI DI FISICA
}

per ORESTE MURANI.

Manuali Hoepli, Milnno 1897.

I maggiori pregi del libro sono la spigliatezza delle linee generali, la ricchezza di larsi chiare e scultorie, semplici oppure eleganti, l’abondanza degli esempii piu famigliari agli 
alunni che hanno una coltura media, come quelli delle scuole secondarie superiori; la parsimonia nell' uso della matematica elementarissima, e nella scelta e trattazione di alcuni argomenti - come pure il fermarsi e l'insistere su que' punti, la cui importanza sfuggirebbe a un lettore novizio.

Il libro del Prof. Murani sarà darvero vantaggioso aggli studenti delle scuole secondarie superiori, e a chi ruole acquistare alcune positive cogniziori intorno al progresso della fisica; inoltre la lettura del rolumetto puo arviare il pensiero dell' insegnante a delimitare con rapiditá una lezione elementare di fisica, come gli suggerirebbe in una geniale courelsazione l'Autore - un conferenziere che, si è formato sul Tyndall, un antico allievo dell' Ateneo pisano, un professore che conta ormai una lunga carriera didattica nei principali Istituti tecnici, dove non mancano grli incoragggiamenti ad insegnare la fisica con intelletto d'amore.

Ma il volumetto presenta agli insegnanti un problema: conviene adottarlo come libro di testo?

Gli studenti vi potranno studiare « come il largo riassunto le cose viste e udite nella scuola, ed io sono convinto che il libro di testo debba essere specialmente per la fisica, cosi multiforme e distrattiva, un largo riassunto anzichè un mare magnum; peró il testo non puó tacẹre di alcuni argomenti, che vanno trattati con metodo, specialmente nelle scuole liceali, dove la fisica recherà sempre un largo contributo nello svolgimento delle facoltà psichiche degli alunni. Perchè l'A. non ha riassunto i suoi stessi «Elementi di meccanica » editi dal Vallardi, con quella larghezza ch' egli tiene nelle lezioni alla sezione di ragioneria e commercio dell Istituto tecnico, nella quale sezione la fisica e materia di coltura come in licen?

Credo appormi bene pensando che il l'rof. Murani nel ristampare - come non dubito che avrí presto occasione - il suo libro, non si limiterà a toglierne qualche traccia di estensione affrettata, ma si diffonderà assai piu in tutta la meccanica e nel calore facendo uscire due desideratissimi volumetti, della mole dell' ottica o dell' elettricita che da sole occupano i. due terzi della presente publicazione. Allora, l'A vorri certo far posto anche a quelle nozioni di cosmografia che sono

Serie $I V$, Vol. $V^{*}$. 
incluse nei programmi liceali. Se poi intenderà ancora cularsi di quelli che non seguono corsi regolari di fisica e che non voramno essere dimenticati dopo aver approfittato di questi «elementi »; il Prol. Murani non stimerà indiscreta la preghiera che egli voglia spigolare dal bel corso che fia al politecnico di Milano qualche succosa pagina d'ottica fisica, dedicare un capitoletto agli effetfi fisiologici dell' elettricita, invece di brevi e sparsi cenni, dar maggiore risalto agli ultimi studii sulle soluzioni, sui vapori, sulla elettrolisi e polarizazione degli elettrodi; e fare anche una esposizione, direi, d'indole piti storica, su alcuni argomenti che sono tuttora in viva di. scussione, quali oggi sarebbero i raggi di Röntgen, le scariche fulminee, le oscillazioni Hertziane.

A. Rovida.

\section{RIVISTA}

\section{Journal de Physique.}

Serie III, tomo 5, Ottobre, 1896.

Lippmans G. Sul mantenimento del moto del pendolo senza perturbazioni (pp. 429-434). - La soppressione delle perturbazioni a cui va soggetto il moto d' un pendolo attaccato ad un orologio è possibile, e l'A., con alcune considerazioni teoriche, giunge alla conclusione che: Se, passando per una stessa posizione, il pendolo riceve due impulsi successivi uguali nel salire e nello scendere, la somma algebrica delle perturbazioni prodotte $\dot{e}$ rigorosamente nulla. Descrive quindi una disposizione elettrica per la quale si possono dare degli impulsi istantanei ad un pen. dolo in un determiunto punto del suo cammino; disposizione nella quale si utilizzano non correnti in circuito chiuso, ma cariche e scariche d'elettricita statica; quindi $\dot{\theta}$ indipendente dalla resistenza del circuito e dalla qualitá dei contatti. L'A. accenna anche una disposizione semplicizzata, peró meno perfetta.

WEIBS P. Ricerche sulla calamitazione della magnetite cristallizzata (pp. 435-453). - L'A ricerca l'influenza dell' orientazione delle particelle elementari della magnetite cristallizzata sull'altro fenomeno d'orientazione che è probabilmente la calamitazione; e studia gli ottaedri $\theta$ i dodecaedri, nei quali fin da principio vede che la magnetizzazione non $\dot{e}$ la stessa in diverse direzioni rispetto argli assi cristallografici. Adopra, modificandolo, il metodo 